\title{
Secretion of Translationally Controlled Tumor Protein/Histamine Releasing Factor and Its Relevance to Parasitic Infections
}

\section{Maeng $\mathbf{J}$ and Lee $\mathrm{K}^{*}$}

Graduate School of Pharmaceutical Sciences, College of Pharmacy, Ewha Womans University, Seoul 120-750, Republic of Korea

\begin{abstract}
Allergic diseases, including atopic dermatitis, allergic rhinitis, and asthma, develop when the immune system is hypersensitized by specific allergens. Translationally controlled tumor protein (TCTP), also known histaminereleasing factor (HRF) because of its cytokine-like activity, mediates late phase reaction leading to allergy and chronic inflammatory diseases in the human. TCTP exhibits HRF activity, when it is released from the cells and becomes dimerized under the inflammatory conditions. It has been shown that TCTP is secreted from cells by a TSAP6-mediated, exosomal route, and exported by H,K-ATPase-mediated process. TCTP is released from various parasitic organisms during parasitic infections. Secreted TCTP is implicated in allergic immune responses to parasites, in the pathogenesis of parasitic infections and also in the evasion of host's immune activity for parasite survival. This review, briefly collates the current information on the secretion of TCTP/HRF by parasitic species and the biological and clinical implications of such release in parasitic diseases such as malaria.
\end{abstract}

Keywords: Allergy; Histamine releasing factor (HRF); Immune response; Parasites; Secretion; Translationally controlled tumor protein (TCTP)

\section{Introduction}

Translationally controlled tumor protein (TCTP), found both intracellular and extracellular milieu of cells, is expressed in most of organisms, including plants, animals and humans in which it is highly conserved (reviewed in [1]). TCTP plays a multitude of biological roles in cellular processes, for example, the regulation of cell cycle [2], apoptosis [3], and malignant transformation [4]. Extracellular TCTP has the cytokine-like histamine releasing activities (HRA) [5,6].

Since the identification of TCTP as a histamine releasing factor (HRF) [6], a great body of evidence has accumulated on the involvement of TCTP/HRF in the pathophysiology of allergic diseases [7-11]. An interesting discovery in this respect is that dimerization of TCTP is critical for its cytokine-like functions and promotion of the release of mediators in allergic responses [5]. TCTP is found in the extracellular milieu, when exported from the cells via endoplasmic reticulum (ER)/Golgi-independent, nonclassical pathway involving exosomal exit, mediated by TSAP6 [12]. We later, showed that $\mathrm{H}, \mathrm{K}$ ATPase is involved in the secretion of TCTP [13] and underscored the need for further investigation to define the exact mode of TCTP's cellular export.

Once secreted and activated TCTP/HRF on binding with its receptor, TCTP triggers the release of histamine [6], IL-4, and IL13 from basophils [14], IL-8 from eosinophils [15], and IL-8 and granulocyte/macrophage colony-stimulating factor (GM-CSF) from human bronchial epithelial cells [16], and promotes the B cell growth [17].

In clinical situations, dimerized TCTP/HRF was detected in extracellular biological fluids from asthma and atopic patients [5]. Also, TCTP/HRF was found in the cutaneous blisters from allergic patients [11], and bronchoalveolar lavage (BAL) fluids from eosinophilic pneumonia patients [16]. Involvement of TCTP/HRF in various allergic diseases such as ragweed-allergic rhinitis [18], asthma and rhinitis [19], and food allergies and dermatitis [20] has been indicated. Importantly, inhibition of the secretion of TCTP [13], and/or blocking agents for dimerized TCTP [21], was shown to be effective in alleviating the inflammatory symptoms in vivo by our researches.

Parasites also actively secrete TCTP homologs [22-27] and they are readily detected in the biological fluids of infected hosts. TCTP/ HRF homologs of parasitic origins also showed conservation of TCTP among various species. They play pivotal role in evading the host immune responses and in the development of parasitic diseases in host organisms [22-27]. This review will attempt to highlight the export mechanism for TCTP and the role of the secreted TCTP/HRF in parasitic species (Figure 1).

\section{Secretion and functions of TCTP -general}

The cellular export of TCTP has been demonstrated in leukemic and erythroid cells [12], and non-immune bronchial epithelial cells [16]. But how exactly TCTP is secreted from the cells is not fully understood. While a majority of proteins exported out of cells contains signal sequences and their export follows the classical ER/Golgimediated secretory pathway, TCTP which has no signal peptide, is believed to utilize a non-classical pathway, mediated by exosomal route [12]. TSAP6, a p53-responsive transmembrane molecule, is reported to facilitate the export of leaderless TCTP by promoting the exosomal transport of TCTP-laden exosomes [12], as confirmed in the TSAP6/ Steap3-null mice [12,22].

In addition, our group suggested that the transport of TCTP occurs via H,K-ATPase, because proton pump inhibitors (PPIs), for instance, pantoprazole and omeprazole inhibit the secretion of TCTP from the cells. Inhibition of TCTP secretion by PPI ameliorates allergic

*Corresponding author: Lee K, Graduate School of Pharmaceutical Sciences, College of Pharmacy, Ewha Womans University, Seoul, Korea, Tel: +82 3277 3024; E-mail: klyoon@ewha.ac.kr

Received June 05, 2014; Accepted July 30, 2014; Published August 07, 2014

Citation: Maeng J, Lee K (2014) Secretion of Translationally Controlled Tumor Protein/Histamine Releasing Factor and Its Relevance to Parasitic Infections. Pharm Anal Acta 5: 304. doi:10.4172/2153-2435.1000304

Copyright: @ 2014 Maeng J, et al. This is an open-access article distributed under the terms of the Creative Commons Attribution License, which permits unrestricted use, distribution, and reproduction in any medium, provided the original author and source are credited. 


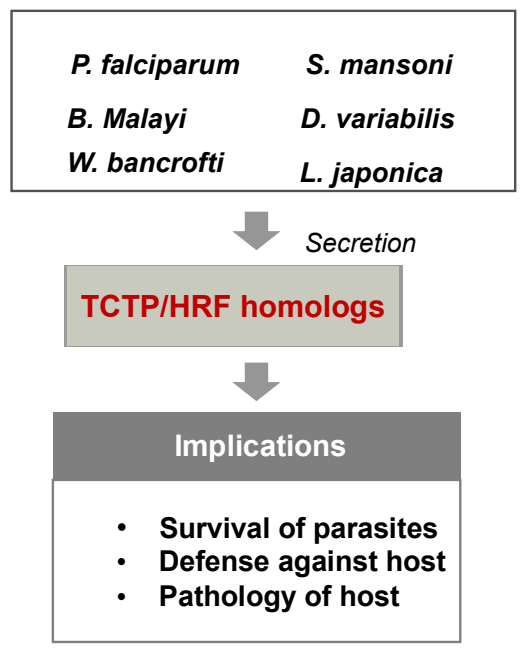

Figure 1: A Schematic diagram depicting the scope of this review. TCTP homologues are actively secreted during various parasitic infections (malaria, filariasis, ixodid tick, lamprey, and Schistosoma among others) and they are readily detected in the biological fluids of infected hosts. Parasitic homologues of TCTP are involved in histamine release, eosinophilic infiltration, and $\mathrm{B}$ cell regulation, all, possibly involved in the manifestations of pathologic symptoms in the host and in the modulation of host-immune functions. In addition, anti-oxidant- and heat shock protein (HSP)-like functions of parasitic TCTPs also seem to contribute to the survival of parasites against host surveillance responses during the parasitic infection. Overall, parasitic TCTPs play a pivotal roles in evading the host immune responses and in the pathogenesis of parasitic diseases in host organisms [25-30].

symptoms in vivo [13], suggesting that inhibition of TCTP release is a logical approach in the therapeutics for allergic inflammation. We suggested that TCTP secretion via H,K-ATPase is achieved through direct or indirect regulation of exosomal exit, endocytosis, and intracellular calcium contents. This suggestion awaits verification [13].

Factors known to facilitate cellular export and secretion of TCTP include macrophage-colony stimulating factor (M-CSF) [23], oxidative agents like hydrogen peroxide $\left(\mathrm{H}_{2} \mathrm{O}_{2}\right)$ [16], and dioxin [24]. The reported ability of hydrogen peroxide to induce the secretion of TCTP by human bronchial epithelial cells supports the speculation that oxidative environments produced by allergic phenomena provoke the release of TCTP to mediate the allergic responses in airway inflammation [16].

\section{Secretion and Functions of TCTP in Parasites}

Conservation of the tertiary structure and functions of TCTP through evolution, suggests that the biological functions of extracellular TCTP of mammals are shared by parasites including Plasmodium falciparum, Wuchereria bancrofti, Brugia malayi, Schistosoma mansoni, Lampetra japonica and Dermacentor variabilis, all of which excrete TCTP that is regarded to be immunogenic into its vertebrate host [25-30].

\section{Plasmodium falciparum}

The wide spread of Plasmodium falciparum, worldwide in humans, results in malaria exhibiting the variety in clinical manifestations and causes over 2 million deaths yearly (reviewed in [31]). Release of proinflammatory mediators during the allergic inflammation in the host is the principal event in the manifestations of malaria [31]. Malarial TCTP is found in the sera of infected patients [26] and exhibits cytokine-like activities [26,32]. Plasmodium TCTP is linked with elevated basophil reactivity in vivo, suggesting the role of malarial TCTP in the allergenic inflammatory symptom in malaria patients [32].

MacDonald and colleagues [26] were the first to characterize a functional homologue of Plasmodium falciparum TCTP (PfTCTP) [26]. PfTCTP is secreted at a high level into serum from malaria patients [26]. Recombinant PfTCTP also binds to calcium [33], like the other TCTP homologs, and potently provokes histamine secretion from human basophils, and IL-8 from eosinophils [26]. PfTCTP in human plasma affects immune response during malaria and is high enough to generate eosinophil and basophil response in the host [26].

However, this cytokine-like activity of malarial TCTP is found to exhibit lower activity than that of its human homolog (HsTCTP) [26]. This observation was recently corroborated by the finding that PfTCTP has less B cell-proliferating effect than HsTCTP, while PfTCTP exhibits a stronger affinity for B cell [34]. These results indicate that plasmodial TCTP possibly has an inhibitory effect on host immune responses [34]. In this context, subtle structural differences between TCTPs of human and plasmodial origins (e.g. P. knowlesi - PkTCTP), such as the presence of an extra $\alpha$-helix in the latter [35], were invoked to explain the differences in their activities. It was suggested that Plasmodial TCTP may act as a dominant negative mutant, thereby hindering the normal host immunity [35].

Malarial TCTP is present in the plasmas of both from lightly and heavily affected individuals during malarial infection [26]. It is speculated that plasma concentration of PfTCTP may reflect the total burden of parasites rather than that circulating in the blood as majority of malarial organisms is sequestered in vessels of internal organs [26] and not seen dispersed in the circulation or systemically. Therefore, it may be reasonable to speculate that secretion of PfTCTP by parasites to be occurs locally rather than systemically and that profound TCTP secretion by parasites in localized regions probably influences immune responses by eosinophils and basophils during parasitic infections. In another perspective, it is suggested that histamine promotes parasite survival by inhibiting the potential blood coagulation of disseminated vasculatures, and by assisting the parasite sequestration through the facilitation of endothelial adhesion $[26,32,35]$.

In clinical settings, emergence of widespread resistance to antimalarial drugs is a matter of great concern. The anti-malarial drug, artemisinin, binds to rodent malarial pathogen $P$. yoelii TCTP (PyTCTP) and resistance to artemisinin correlated with the expression of PyTCTP [36]. Artemisinin-resistant $P$. yoelii, expresses elevated TCTP levels, compared to artemisinin-sensitive species [36]. Reactivity of $\left[{ }^{3} \mathrm{H}\right]$ dihydroartemisinin with both monomeric and dimeric TCTP in parasites, indicates the functions of TCTP dimer in the artemisinin action [36]. A recent study elaborated the defined mode of artemisinin interaction with plasmodial TCTP [37]. Using bioinformatic approaches, surface plasmon resonance spectroscopy, and mass spectrometry, these authors verified novel interaction sites of artemisinin with TCTP. They are in close vicinity to amino acid residues 19-46, 108-134 and 140-163 which are related to important functions of TCTP and their interaction with TCTP was implicated in the antimalarial effect of artemisinin [37]. To sum up, malarial TCTP seems to be involved not only in the immunopathogenesis during malaria but also in the development of drug resistance

\section{Brugia malayi and Wuchereria bancrofti}

TCTP homologs from filarial parasitic worms including Brugia malayi (BmTCTP) and Wuchereria bancrofti (WbTCTP) were assumed 
to modulate allergic inflammatory responses during filarial infections [25]. Both the helminthes are primary causes of human lymphatic filariasis that causes significant morbidity in humans, especially in the tropic areas [38]. Human lymphatic filariasis from B. malayi $(\mathrm{Bm})$ and $W$. bancrofti $(\mathrm{Wb})$ resembles the allergic inflammatory responses, such as the hyperreactivity immune responses, pulmonary allergic symptoms, and eosinophilic infiltration [39].

It is found that significant amounts of BmTCTP are detected in the excretion and secretion products from microfilaria though it lacks apparent leader sequence [25]. Interestingly, both $\mathrm{Bm}$ and WbTCTP have calcium-binding properties and are potent inducers of the histamine release from murine mast cells and basophils, and eosinophilic infiltration into the peritoneal cavity of mice [25]. This indicates the pivotal role of filarial TCTP in the development of allergic inflammation and immunopathogenesis in filariasis by TCTPprovoked histamine release [25].

As observed in P. falciparum [40], expression of BmTCTP is regulated along with the filarial stages, suggesting the contribution of TCTP control in the adaptation of parasites to changing host environment [25]. Also, as with malarial [26], rat, and human versions of TCTP [5,41], filarial BmTCTP is capable of self-interaction to form multimers of TCTP in solution via Lupas coiled coil structure [25]. Consistent with our observation on the dimerization of TCTP in rat and human TCTPs [5,41] through the disulfide bond [5], BmTCTP also appears to form disulfide linkage between TCTP monomers [25].

Another interesting feature for BmTCTP, is that it has an antioxidant function protecting the parasites from oxidative damage, mediated by its cysteine residues [42]. Therefore, TCTP is proposed as a non-classical antioxidative protein in the filarial organisms enabling them to counteract the deleterious effects of oxygen radicals produced by the host [42]. Because BmTCTP is present both in intracellular and extracellular milieus, BmTCTP offers a protective mechanism for survival of the parasite against both endogenous and exogenous oxidative damages [25,42].

\section{Schistosoma mansoni}

The trematode, Schistosoma mansoni, causes schistosomiasis, which is transmitted through water, and infects the urinary tract and intestines of human, causing eosinophilia and plasma histamine elevation [43]. TCTP from Schistosoma mansoni (SmTCTP) is abundantly secreted into host, in spite of its lack of signal sequences [29]. SmTCTP is detected in the excretory-secretory (ES) product of schistosomula of S. mansoni in a multrimeric form [29]. Also, differential expression of TCTP during life cycle progression is found in S. mansoni [29].

Similar to filarial TCTP, SmTCTP also exhibits calcium binding function, mediates histamine secretion from both basophils and mast cells, and accumulates eosinophils in the peritoneal cavity of mice, indicating its pathogenic relevance in the allergic inflammation in schistosomiasis [29]. Since SmTCTP is a potent antigen that can promote the histamine release into the environment surrounding the parasite, migrating schistosomula may exploit this fact for disseminating within host [29]. For instance, histamine released by SmTCTP is thought to induce the dilatation of vasculature, which helps the migration of the parasites [44].

Both HsTCTP and SmTCTP showed heat shock protein (HSP)like properties and regarded as molecular chaperones that prevent the noxious effect from heat-induced protein denaturation, through the association with various denatured proteins [45]. It was speculated that SmTCTP can act as a small molecular weight HSP and that heat-induced TCTP protects the parasites from thermal shock from the warm-blooded host environment [45]. In addition to plasmodial TCTP, SmTCTP is also suggested as a potential target for artemisinin derivatives in schistosomiasis, because anti-malarial artemisinin derivatives also revealed to be effective in schistosomiatic illness [46].

\section{Dermacentor variabilis}

Histamine is significantly released at the tick-attached regions of mammalian hosts and inhibits the attachment and feeding of ticks $[47,48]$. In addition, histamine-mediated inflammation is one of the host defense mechanisms against tissue injury by feeders $[47,48]$.

It was reported that TCTP homologues of ixodid tick which lack signal peptides, are secreted in the tick saliva and get injected into the host at the time of tick feeding $[27,28]$. The American dog tick, Dermacentor variabilis (Dv) TCTP is apparently secreted into saliva, hemolymph of tick, and into extracellular milieu [28]. DvTCTP and other ixodid tick orthologs are highly conserved and at least four ixodid tick species are shown to be capable of secreting TCTP into culture medium [27].

Recombinant DvTCTP and salivary gland extract containing DvTCTP were shown to provoke histamine release from rat basophils [28]. It is suggested that secreted DvTCTP may stimulate the histamine release from basophil at the feeding site, to assist the tick feeding by increasing the blood flow [28]. Histamine secretion by DvTCTP is also thought to modulate host immune responses [28]. Universal expression and high conservation of ixodid ticks, including DvTCTP, makes TCTP homologues attractive target antigens in the development of general tick control strategies.

\section{Lampetra japonica}

More recently, a novel TCTP homolog was cloned from buccal gland of Lampreys [30]. Lampreys, ancient living jawless vertebrates, are parasitic to fishes, and their buccal gland secretions are known to inhibit blood coagulation (reviewed in [49]). Immunoblot analysis revealed that the Lampetra japonica TCTP (LjTCTP) is secreted from the buccal gland of L. japonica [30] probably through a non-classical route, as proposed earlier [12].

Studies with recombinant LjTCTP also demonstrated the histamine-releasing ability from rat basophils [30]. It is indicated that LjTCTP-induced histamine release may mediate the anticoagulant effect by expressing the tissue anticoagulant, thrombomodulin, at the surface of endothelium [35]. Because histamine causes vasodilation and inflammation in the host, secreted LjTCTP can be assumed to regulate the immune reaction in the lamprey [30].

\section{Relevance of TCTP Secretion in Parasitic infections}

Large amounts of leaderless TCTP homologues of human [6], rodent [23], and diverse parasites including Plasmodium falciparum, Wuchereria bancrofti, Brugia malayi, Schistosoma mansoni, Lampetra japonica and Dermacentor variabilis, are secreted into the extracellular milieu of host's biological fluids, causing manifestations of allergic responses [25-30].

In patients, these TCTP homologs are released to the microenvironment contributing to the pathogenesis and virulence of parasitic diseases. These effects are preceded by histamine release from basophils or mast cells [25-30], infiltration of eosinophils through chemotaxis [25,29], B-cell mediated activity [34] and inflammation 
Citation: Maeng J, Lee K (2014) Secretion of Translationally Controlled Tumor Protein/Histamine Releasing Factor and Its Relevance to Parasitic Infections. Pharm Anal Acta 5: 304. doi:10.4172/2153-2435.1000304

in the host. Given that the parasitic TCTPs can induce eosinophil infiltration and histamine secretion from mast cells or basophils, they become critical players in the pathogenesis of parasitic diseases.

The mechanism for parasite defense against host attack is known to involve shedding of pathogenic antigens, and modulation of immune reaction of host [50,51]. Parasitic TCTPs are suggested to modulate the host immune reaction through the mechanism such as histamine release and the regulation of B cell reactivity [34]. Studies on crossreactivities using recombinant $S$. japonicum, Clonorchis sinensis and rat TCTP indicated that antibodies against anti-parasitic TCTP may reduce the sensitivities of pathogen-induced immune reactivity by host [52]. In addition, as found with mammalian TCTPs, heat shock protein-like properties [45] and anti-oxidant functions [42] of TCTP homologs may influence the survival of the parasites in the microenvironment of host.

Parasitic TCTPs is believed to aid the spread of organisms within host via the pathogen survival mechanisms and immune-evasive strategy. It was speculated that release of histamine, a vasodilator, by PfTCTP, during malarial infection might dilate the vasculature to assist the vascular circulation of the parasites [26]. As speculated, histamine may inhibit the blood coagulation and support the sequestration of parasites, possibly through expression of anticoagulants and sequestration receptors, and promotion of endothelial adhesion $[26,32,35]$. Detailed mechanisms underlying the effects of TCTP homologs on host immune regulation and the pathogenesis of parasitic illness need to be studied.

Taken together, this review shows that most parasitic TCTPs may participate in promoting pathophysiologic processes in an infected host as well as in their beneficial survival strategies to sustain a life against the host defense system. Parasitic homologs of TCTP may be a potential target in the parasitic disease such as malaria, filariasis, and schistosomiasis.

\section{Conclusion}

Parasitic TCTPs are central players both in host pathology and survival of the pathogens. Parasitic homologs of TCTP, conserved through phylogeny, are secreted into the fluids of vertebrate host. Secreted TCTPs mediate late phase immune responses that contribute to the immunopathology and pathogenesis of parasites diseases. Differences between human and parasitic TCTPs may contribute to parasite survival. Elucidation on the secretion mechanism and the pathogenesis of TCTP homologs, and on the nature of the receptors that TCTP homologs bind to, may offer new approaches to the prevention and therapy of parasitic diseases.

\section{References}

1. Bommer UA, Thiele BJ (2004) The translationally controlled tumour protein (TCTP). See comment in PubMed Commons below Int J Biochem Cell Biol 36: $379-385$

2. Gachet Y, Tournier S, Lee M, Lazaris-Karatzas A, Poulton T, et al. (1999) The growth-related, translationally controlled protein $\mathrm{P} 23$ has properties of a tubulin binding protein and associates transiently with microtubules during the cell cycle. J Cell Sci 112 ( Pt 8): 1257-1271.

3. Li F, Zhang D, Fujise K (2001) Characterization of fortilin, a novel antiapoptotic protein. See comment in PubMed Commons below J Biol Chem 276: 4754247549 .

4. Tuynder M, Susini L, Prieur S, Besse S, Fiucci G, et al. (2002) Biological models and genes of tumor reversion: cellular reprogramming through tpt1/ TCTP and SIAH-1. See comment in PubMed Commons below Proc Natl Acad Sci U S A 99: 14976-14981.
5. Kim M, Min HJ, Won HY, Park $\mathrm{H}$, Lee JC et al. (2009) Dimerization of translationally controlled tumor protein is essential for its cytokine-like activity. See comment in PubMed Commons below PLoS One 4: e6464.

6. MacDonald SM, Rafnar T, Langdon J, Lichtenstein LM (1995) Molecula identification of an IgE-dependent histamine-releasing factor. See comment in PubMed Commons below Science 269: 688-690.

7. Lichtenstein LM (1988) Histamine-releasing factors and IgE heterogeneity. See comment in PubMed Commons below J Allergy Clin Immunol 81: 814-820.

8. Liu MC, Proud D, Lichtenstein LM, MacGlashan DW Jr, Schleimer RP, et al. (1986) Human lung macrophage-derived histamine-releasing activity is due to IgE-dependent factors. See comment in PubMed Commons below J Immunol 136: $2588-2595$.

9. MacDonald SM, Lichtenstein LM, Proud D, Plaut M, Naclerio RM, et al. (1987) Studies of IgE-dependent histamine releasing factors: heterogeneity of IgE. See comment in PubMed Commons below J Immunol 139: 506-512.

10. Pasmans SG, Witteman AM, Aalbers M, Boonstra JG, Mul EP, et al. (1994) Variability of IgE-dependent histamine-releasing activity in supernatants of human mononuclear cells. See comment in PubMed Commons below Int Arch Allergy Immunol 103: 44-52.

11. Warner JA, Pienkowski MM, Plaut M, Norman PS, Lichtenstein LM (1986) Identification of histamine releasing factor(s) in the late phase of cutaneous IgE-mediated reactions. See comment in PubMed Commons below J Immunol 136: 2583-2587

12. Amzallag N, Passer BJ, Allanic D, Segura E, Thery C, et al. (2004) TSAP6 facilitates the secretion of translationally controlled tumor protein/histaminereleasing factor via a nonclassical pathway. J Biol Chem 279: 46104-46112.

13. Choi S, Min HJ, Kim M, Hwang ES, Lee K (2009) Proton pump inhibitors exert anti-allergic effects by reducing TCTP secretion. See comment in PubMed Commons below PLoS One 4: e5732.

14. Schroeder JT, Lichtenstein LM, MacDonald SM (1997) Recombinant histaminereleasing factor enhances IgE-dependent IL-4 and IL-13 secretion by human basophils. See comment in PubMed Commons below J Immunol 159: 447-452.

15. Bheekha-Escura R, MacGlashan DW, Langdon JM, MacDonald SM (2000) Human recombinant histamine-releasing factor activates human eosinophils and the eosinophilic cell line, AML14-3D10. See comment in PubMed Commons below Blood 96: 2191-2198.

16. Yoneda K, Rokutan K, Nakamura Y, Yanagawa H, Kondo-Teshima S, et al. (2004) Stimulation of human bronchial epithelial cells by lgE-dependent histamine-releasing factor. See comment in PubMed Commons below Am J Physiol Lung Cell Mol Physiol 286: L174-181.

17. Kang HS, Lee MJ, Song H, Han SH, Kim YM, et al. (2001) Molecular identification of IgE-dependent histamine-releasing factor as a B cell growth factor. See comment in PubMed Commons below J Immunol 166: 6545-6554.

18. Brunet C, Bédard PM, Lavoie A, Jobin M, Hébert J (1992) Allergic rhinitis to ragweed pollen. II. Modulation of histamine-releasing factor production by specific immunotherapy. See comment in PubMed Commons below J Allergy Clin Immunol 89: 87-94.

19. Pasmans SG, Aalbers M, van der Veen MJ, Knol EF, van der Zee JS, et al (1996) Reactivity to IgE-dependent histamine-releasing activity in asthma or rhinitis. See comment in PubMed Commons below Am J Respir Crit Care Med 154: 318-323.

20. Sampson HA, Broadbent KR, Bernhisel-Broadbent J (1989) Spontaneous release of histamine from basophils and histamine-releasing factor in patients with atopic dermatitis and food hypersensitivity. See comment in PubMed Commons below N Engl J Med 321: 228-232.

21. Kim M, Chung J, Lee C, Jung J, Kwon Y, et al. (2011) A peptide binding to dimerized translationally controlled tumor protein modulates allergic reactions. See comment in PubMed Commons below J Mol Med (Berl) 89: 603-610.

22. Lespagnol A, Duflaut D, Beekman C, Blanc L, Fiucci G, et al. (2008) Exosome secretion, including the DNA damage-induced p53-dependent secretory pathway, is severely compromised in TSAP6/Steap3-null mice. See comment in PubMed Commons below Cell Death Differ 15: 1723-1733.

23. Teshima S, Rokutan K, Nikawa T, Kishi K (1998) Macrophage colonystimulating factor stimulates synthesis and secretion of a mouse homolog of a human IgE-dependent histamine-releasing factor by macrophages in vitro and 
Citation: Maeng J, Lee K (2014) Secretion of Translationally Controlled Tumor Protein/Histamine Releasing Factor and Its Relevance to Parasitic Infections. Pharm Anal Acta 5: 304. doi:10.4172/2153-2435.1000304

in vivo. See comment in PubMed Commons below J Immunol 161: 6356-6366.

24. Oikawa K, Ohbayashi T, Mimura J, Fujii-Kuriyama Y, Teshima S, et al. (2002) Dioxin stimulates synthesis and secretion of IgE-dependent histaminereleasing factor. See comment in PubMed Commons below Biochem Biophys Res Commun 290: 984-987.

25. Gnanasekar M, Rao KV, Chen L, Narayanan RB, Geetha M, et al. (2002) Molecular characterization of a calcium binding translationally controlled tumor protein homologue from the filarial parasites Brugia malayi and Wuchereria bancrofti. See comment in PubMed Commons below Mol Biochem Parasitol 121: $107-118$.

26. MacDonald SM, Bhisutthibhan J, Shapiro TA, Rogerson SJ, Taylor TE, et al. (2001) Immune mimicry in malaria: Plasmodium falciparum secretes functional histamine-releasing factor homolog in vitro and in vivo. See comment in PubMed Commons below Proc Natl Acad Sci U S A 98: 10829-10832.

27. Mulenga A, Azad AF (2005) The molecular and biological analysis of ixodid ticks histamine release factors. See comment in PubMed Commons below Exp Appl Acarol 37: 215-229.

28. Mulenga A, Macaluso KR, Simser JA, Azad AF (2003) The American dog tick, Dermacentor variabilis, encodes a functional histamine release factor homolog. See comment in PubMed Commons below Insect Biochem Mol Biol 33: 911 919.

29. Rao KV, Chen L, Gnanasekar M, Ramaswamy K (2002) Cloning and characterization of a calcium-binding, histamine-releasing protein from Schistosoma mansoni. See comment in PubMed Commons below J Biol Chem 277: 31207-31213.

30. Sun J, Wu Y, Wang J, Ma F, Liu X, et al. (2008) Novel translationally controlled tumor protein homologue in the buccal gland secretion of Lampetra japonica. See comment in PubMed Commons below Biochimie 90: 1760-1768.

31. Mecheri S (2012) Contribution of allergic inflammatory response to the pathogenesis of malaria disease. See comment in PubMed Commons below Biochim Biophys Acta 1822: 49-56.

32. Pelleau S, Diop S, Dia Badiane M, Vitte J, Beguin P, et al. (2012) Enhanced basophil reactivities during severe malaria and their relationship with the Plasmodium falciparum histamine-releasing factor translationally controlled tumor protein. See comment in PubMed Commons below Infect Immun 80: 2963-2970.

33. Bhisutthibhan J, Philbert MA, Fujioka H, Aikawa M, Meshnick SR (1999) The Plasmodium falciparum translationally controlled tumor protein: subcellular localization and calcium binding. See comment in PubMed Commons below Eur J Cell Biol 78: 665-670.

34. Calderón-Pérez B, Xoconostle-Cázares $B$, Lira-Carmona $R$, Hernández-Rivas R, Ortega-López J, et al. (2014) The Plasmodium falciparum translationally controlled tumor protein (TCTP) is incorporated more efficiently into B cells than its human homologue. See comment in PubMed Commons below PLoS One 9: e85514.

35. Hirokawa K, Aoki N (1991) Up-regulation of thrombomodulin by activation of histamine $\mathrm{H} 1$-receptors in human umbilical-vein endothelial cells in vitro. See comment in PubMed Commons below Biochem J 276 : 739-743.

36. Walker DJ, Pitsch JL, Peng MM, Robinson BL, Peters W, et al. (2000) Mechanisms of artemisinin resistance in the rodent malaria pathogen Plasmodium yoelii. See comment in PubMed Commons below Antimicrob Agents Chemother 44: 344-347.

37. Eichhorn T, Winter D, Büchele B, Dirdjaja N, Frank M, et al. (2013) Molecular interaction of artemisinin with translationally controlled tumor protein (TCTP) of Plasmodium falciparum. See comment in PubMed Commons below Biochem Pharmacol 85: 38-45.

38. Michael E, Bundy DA, Grenfell BT (1996) Re-assessing the global prevalence and distribution of lymphatic filariasis. See comment in PubMed Commons below Parasitology 112 : 409-428.
39. Ottesen E (1984) Immunopathology of lymphatic filariasis in man. Springer Semin Immunopathol 2: 373-382.

40. Bhisutthibhan J, Pan XQ, Hossler PA, Walker DJ, Yowell CA, et al. (1998) The Plasmodium falciparum translationally controlled tumor protein homolog and its reaction with the antimalarial drug artemisinin. See comment in PubMed Commons below J Biol Chem 273: 16192-16198.

41. Yoon T, Jung J, Kim M, Lee KM, Choi EC, et al. (2000) Identification of the selfinteraction of rat TCTP/lgE-dependent histamine-releasing factor using yeast two-hybrid system. See comment in PubMed Commons below Arch Biochem Biophys 384: 379-382.

42. Gnanasekar M, Ramaswamy K (2007) Translationally controlled tumor protein of Brugia malayi functions as an antioxidant protein. See comment in PubMed Commons below Parasitol Res 101: 1533-1540.

43. Bruce C, Taylor WH (1996) Plasma histamine in patients with tropical disorders and acute asthma who exhibit eosinophilia. See comment in PubMed Commons below Ann Clin Biochem 33 : 249-252.

44. Gerken SE, Mota-Santos TA, Vaz NM, Correa-Oliveira R, Dias-da-Silva W, et al. (1984) Recovery of schistosomula of Schistosoma mansoni from mouse skin: involvement of mast cells and vasoactive amines. See comment in PubMed Commons below Braz J Med Biol Res 17: 301-307.

45. Gnanasekar M, Dakshinamoorthy G, Ramaswamy K (2009) Translationally controlled tumor protein is a novel heat shock protein with chaperone-like activity. See comment in PubMed Commons below Biochem Biophys Res Commun 386: 333-337.

46. Xiao S, Tanner M, N'Goran EK, Utzinger J, Chollet J, et al. (2002) Recen investigations of artemether, a novel agent for the prevention of schistosomiasis japonica, mansoni and haematobia. See comment in PubMed Commons below Acta Trop 82: 175-181.

47. Kemp DH, Bourne A (1980) Boophilus microplus: the effect of histamine on the attachment of cattle-tick larvae--studies in vivo and in vitro. See comment in PubMed Commons below Parasitology 80: 487-496.

48. Paine SH, Kemp DH, Allen JR (1983) In vitro feeding of Dermacentor anderson (Stiles): effects of histamine and other mediators. See comment in PubMed Commons below Parasitology 86 : 419-428.

49. Xiao R, Pang Y, Li QW (2012) The buccal gland of Lampetra japonica is a source of diverse bioactive proteins. See comment in PubMed Commons below Biochimie 94: 1075-1079.

50. Hofstetter M, Fasano MB, Ottesen EA (1983) Modulation of the host response in human schistosomiasis. IV. Parasite antigen induces release of histamine that inhibits lymphocyte responsiveness in vitro. See comment in PubMed Commons below J Immunol 130: 1376-1380.

51. Lightowlers MW, Rickard MD (1988) Excretory-secretory products of helminth parasites: effects on host immune responses. See comment in PubMed Commons below Parasitology 96 Suppl: S123-166.

52. Chen X, Hu X, Wu Z, Yu X, Ma C, et al. (2007) Immunological cross-reactivity analysis on recombinant histamine-releasing factors from Schistosoma japonicum, Clonorchis sinensis, and Wistar rat. See comment in PubMed Commons below Parasitol Res 100: 749-754. 